\title{
Voices Author Survey
}

In September, the editorial team decided to request feedback from Voices authors about their perceptions of the reviewing process. We were interested to hear whether our attempts at re-focusing on the Voices vision were conspicuous to our authors. In addition, we wanted to know if there were any areas where our processes were ineffective.

Authors from the past two years were emailed with a link to the online survey and 27 responded with answers to most questions. This report presents the results of that survey, affirms the value of our focus on dialogue, and identifies areas where performance has been sporadic.

What is your disciplinary background ie: music therapy, music psychology, sociology, etc?

4 of the authors nominated more than one disciplinary background, but the vast majority of authors (21/27) included music therapy in this list. This shows that we have achieved some degree of diversity, particularly through the Special editions who had invited 3 of the 6 non music therapy publications.

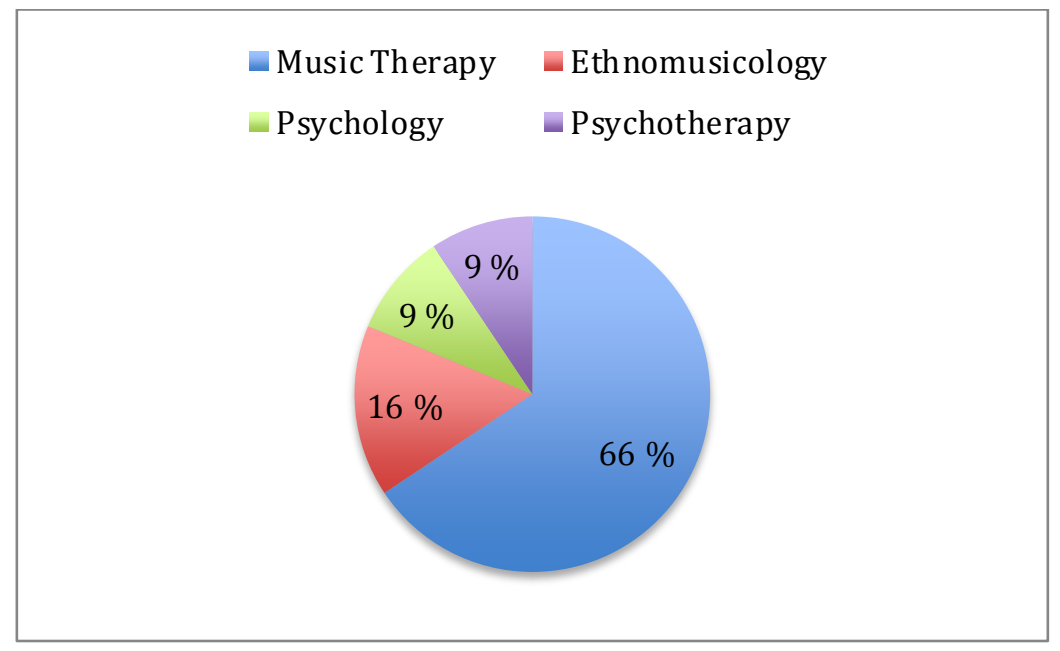

How often do you publish articles ie: once a year, around 3 per year, this is my first attempt?

Of the 27 respondents, there was an even spread of experience across the frequency of authorship. It was conspicuous that this was the first attempt at publication for 7 of the authors, and one noted that she/he had been recommended to Voices for that reason. The final question also asked whether the submission was successful, and the only two who answered 'no' were first time authors. 


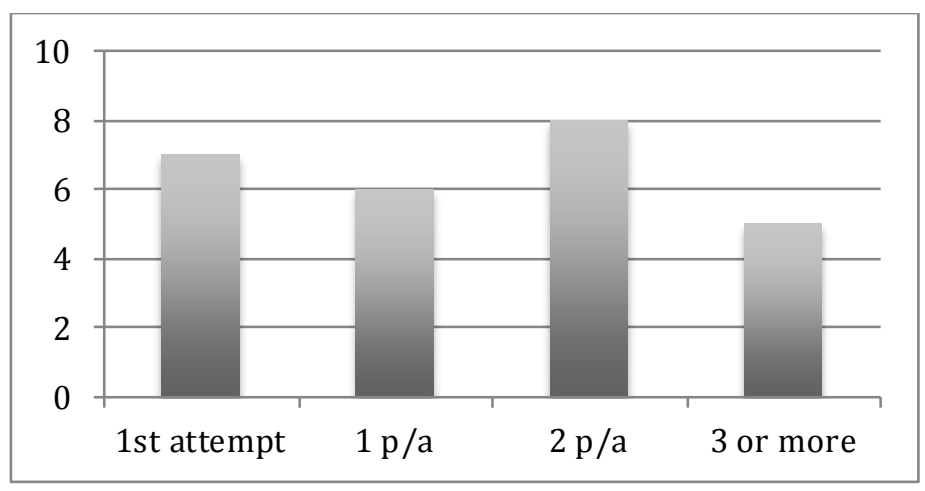

Why did you submit your manuscript to Voices ie: identified with the vision, desire to engage Voices readership, speed of online publishing, etc?

Our focus on clarifying the vision of Voices appears to have been effective and this was the most common answer, with a desire to connect with the Voices readership potentially being considered similarly. Both speed and open access were also a priority for authors, which needs to be more consistently addressed through our processes (see below). A number of authors noted that they had been encouraged or invited by the Editors-in-Chief to submit, mostly for a special edition.

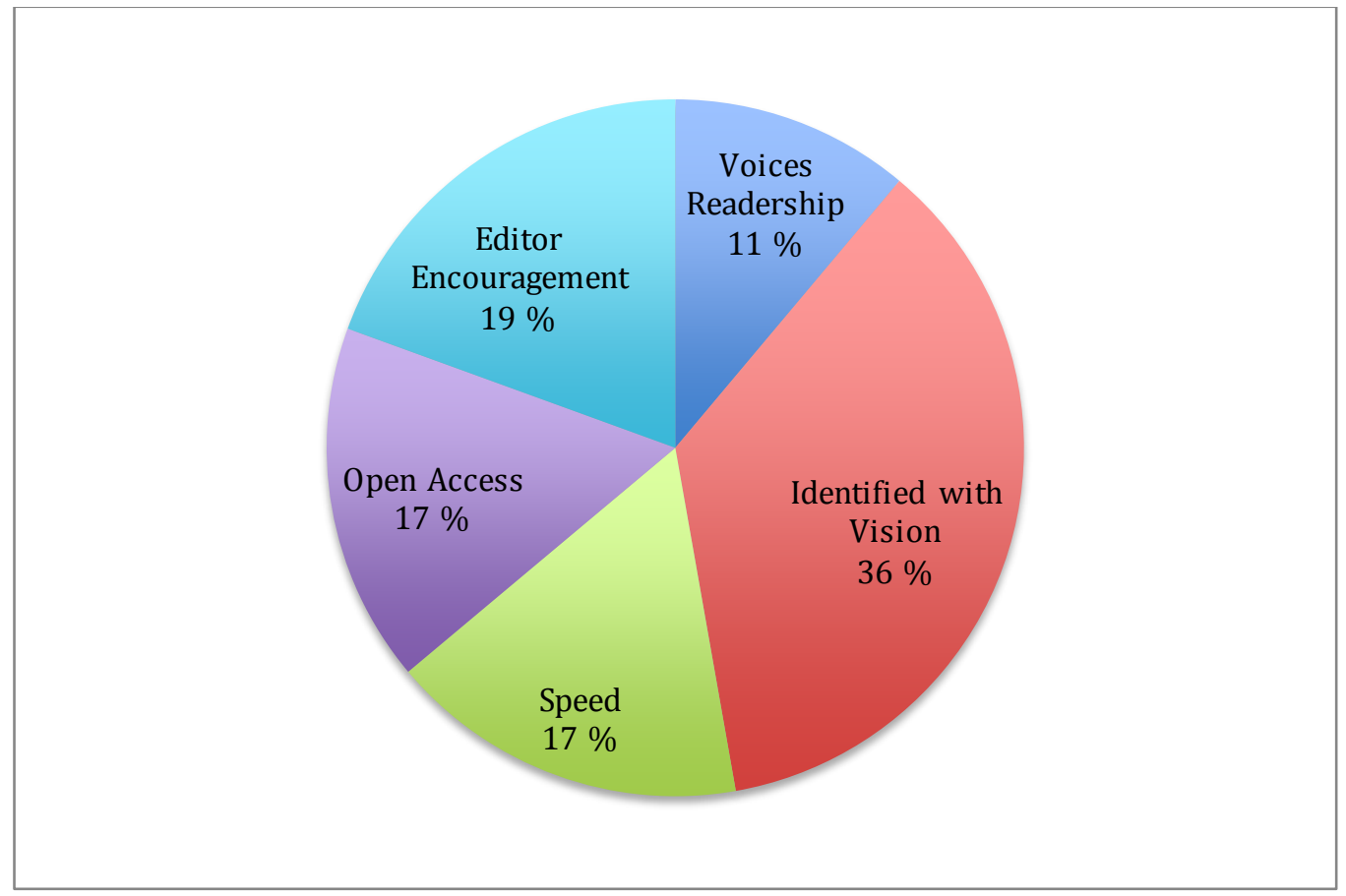

Did you feel the processes were respectful or disrespectful, and in what ways ie: the amount of dialogue, clarity of feedback?

21 of the 27 authors described the processes as respectful, with a number of people sharing lovely feedback such as "there was something refreshing about it, comparatively..." and "one reviewer was blunt, but the editor supported the dialogue respectfully" and "one of the best editors I have worked with." 4 people did not answer this question, one whose paper had been rejected, and noted in another question that they did not feel the content of the Voices publications were always in line with the vision of the journal. Another had experienced an 
extremely long delay in timelines, and the third person did not complete all survey questions. Another three were still waiting on feedback to their submissions. The lack of negative feedback to this question is extremely important for us to acknowledge as a team, particularly because many people have expressed concern about whether this desire for mutual respect was being experienced by authors. Kudos to everyone.

\section{Areas for Improvement}

How were the submission and review processes for you, and were they different to other journals you have experienced, ie: accessibility of website; timeliness of review?

When asked specifically for feedback about the review and submission processes, two areas for improvement were identified. 8 authors felt that the review process was too slow and this will need to be addressed in order for the journal to match the desires of our submitting authors. It is worth noting that the experience of speed was highly variable, with some authors noting how swift and clear the process was, whilst others had the opposite experience. 3 authors also felt that the website was too complex and could be simplified.

What could we do differently as journal editors that would support your manuscript publication ie: more dialogue via email, Skype conversations? The feedback provided about what we could do differently can be categorised into three main areas. The largest category is of authors who are entirely happy with their experience of Voices, and 11 authors did not have any suggestions for improvement.

Faster reviewing. As noted above when authors nominated why they had chosen Voices, pace of the process from submission to publication is a priority. 7 of the authors suggest that this could have been improved, with a small number having experienced extremely long delays. There are a number of levels where delays can occur, and experience suggests that delays occurs at all stages from delegation to editors, identification of willing reviewers, receipt of submissions, dialogue between authors and editors, and final preparation for publication. Whilst we are not likely to be able to perfect this process, there is room for improvement at each stage and we should strive to do so where feasible.

More dialogue. As noted above, experiences were variable but 8 authors also suggested that more dialogue would be helpful. This included one reviewer/author who was not aware of any difference in the reviewing processes between Voices and other journals, even though he/she was aware that there should be. As editors, we are still refining our dialogic strategies and will continue to do so, however this is also an important area for development in our reviewing community and will be the focus of the next few months as we attempt to communicate with those people who volunteered to review for Voices in response to our call 12 months ago. This should continue to be a topic of discussion for us as a team. 